Research Paper

\title{
Analysis of Clinical Target Volume Delineation in Local-regional Failure of Nasopharyngeal Carcinoma after Intensity-modulated Radiotherapy
}

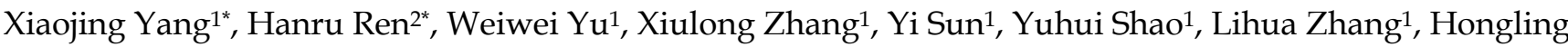 \\ $\mathrm{Li}^{1}$, Xinmiao Yang ${ }^{1}$, Jie $\mathrm{Fu}^{1}{ }^{\circledR}$ \\ 1. Department of Radiation Oncology, Shanghai Jiao Tong University Affiliated Sixth People's Hospital, No. 600, Yishan Road, Shanghai, 200233, China. \\ 2. Department of Orthopedics, Shanghai Pudong Hospital, Fudan University, Pudong Medical Center, Shanghai 201300, P.R China \\ * These authors made equal contributions. \\ $\triangle$ Corresponding author: fujie74@sjtu.edu.cn; Tel: +86-021-24058972; Fax: +86-021-24058972.
}

() The author(s). This is an open access article distributed under the terms of the Creative Commons Attribution License (https://creativecommons.org/licenses/by/4.0/). See http://ivyspring.com/terms for full terms and conditions.

Received: 2019.08.24; Accepted: 2019.12.26; Published: 2020.01.29

\begin{abstract}
OBJECTIVE: To analyze the pattern of local failure in patients with nasopharyngeal carcinoma (NPC) after intensity-modulated radiotherapy (IMRT) and find a more reasonable delineation of the clinical target volume (CTV).

METHODS AND MATERIALS: A total of 212 patients with non-metastatic NPC who underwent IMRT were analyzed. Radiation therapy was run at a total dose of 66-74 Gy (2.0-2.2 Gy fractions). The follow-up of local recurrence and the recurrence-related features were analyzed for the original treatment situation. The failures were delimited as "in-field failure" if $\mathrm{V}_{\text {recur }}$ within the $95 \%$ isodose curve (V95\%) was $\geq 95 \%$; "marginal failure" if V95\% was less than $95 \%$ and not less than $20 \%$; or "out-field failure" if V95\% was $<20 \%$. Kaplan-Meier method was used to calculate the survival rates.

RESULTS: The median follow-up was 43.4 months. The 5 -year local relapse-free survival and overall survival rates were 85.6 and $77.8 \%$, respectively. A total of 18 patients have relapsed. The in-field failure, marginal failure, and out-field failure accounted for $83.3 \%, 11.1 \%$, and $5.6 \%$, respectively. The site of recurrence was basically in the high dose area.

CONCLUSION: These findings suggested that IMRT provide a good local control for patients with NPC, and the in-field failure is the main mode. A wide range of CTV cannot prevent the local recurrence, narrowing the CTV to protect the adjacent organs should be taken into consideration.
\end{abstract}

Key words: Clinical target volume; Delineation; Nasopharyngeal carcinoma; Intensity-modulated radiotherapy

\section{Introduction}

Nasopharyngeal carcinoma (NPC) is the most common cancer of all head and neck malignancies in Southeast Asia. Radiation therapy is regarded as the most effective treatment for NPC [1], and intensity-modulated radiotherapy (IMRT) has been widely used in radiation oncology. Over the past decade, IMRT has been considered to be a major breakthrough in the treatment of NPC due to its ability to offer high radiation doses to targets while preserving adjacent organs $[2,3]$.
However, local recurrence remains to be the leading cause of failure in NPC patients with locally advanced disease [4]. Dawson et al. [5] in the year 2000 are the first to study the connection between recurrence and previous therapeutic dose distribution. After 27 months of follow-up, the results revealed that most of the local recurrence after IMRT treatment was an "in-field" recurrence, which included the gross tumor volume (GTV) and surgical boundary. In the previous years, there are several 
studies on the analogous failure patterns in NPC patients [4, 6-8]. However, Lin et al. [9] have reported that the IMRT used has reduced the volume in NPC patients, and the results showed a similar local control. This suggested the possibility of lowering the clinical target volume (CTV) without affecting the local control rate. NPC patients usually have a longer life expectancy and are more prone to long-term complications due to radiotherapy [10]. The complications such as hearing loss, cranial nerve damage, endocrine dysfunction, and temporal necrosis of temporal lobe that are caused by radiotherapy $[11,12,13,14,15]$ can lead to decreased quality of life in NPC patients [16, 17]. The most significant effort required to ameliorate the outcomes might be an accurate delineation of CTV, which has been a great challenge for radiation oncologists.

Hence, in our study, the initial dose and the local failure pattern of recurrence sites in NPC patients were studied in detailed to present the importance of CTV. The summary of our research revealed that CTV delineation could be further improved to enhance the life quality of NPC patients without reducing local control and long-term survival.

\section{Materials and methods}

\section{Patient characteristics}

A total of 212 patients with NPC who underwent IMRT in our department between September 2008 and December 2018 were retrospectively collected. These NPC patients were newly diagnosed, untreated, and had no distant organ metastasis. The detailed clinical data of patients were presented in Table 1 .

\section{Diagnostic criteria}

All cases received pretreatment assessments involving integral medical history, physical examination, hematology data, Magnetic Resonance Imaging (MRI) scans of the nasopharynx and neck, chest radiographs, and abdominal ultrasound. The retrospective analysis of medical and imaging records of all cases was done according to the American Joint Committee on Cancer (AJCC) version 6 staging system.

\section{Radiotherapy target volume definition}

The ICRU Report Nos. 50 and 62 were referred to define the target. GTV was divided into (1) primary foci GTV (GTV-P) include primary nasopharyngeal tumors and posterior pharyngeal lymph nodes; and (2) lymph nodes GTV (GTV-N) include all cervical metastatic lymph nodes. CTV was divided into high-risk volume (CTV1), which included the high-risk CTV in the nasopharynx and neck. (1) The high-risk CTV of the nasopharynx includes the entire nasopharynx, posterior pharyngeal lymph node area, skull base, slope, parapharyngeal space, pterygopalatine fossa, sphenoid sinus, part of the nasal cavity and $1 / 3$ of the maxillary sinus, and includes the complete GTV; (2) low-risk area CTV2 includes the lymph node area of the neck IV and $\mathrm{Vb}$ areas. There are 4 PTVs in this study: PTV-G (GTV + 5 $\mathrm{mm})$, PTV-N (GTV-N + 5mm), PTV-C1 (CTV1 + 3 $\mathrm{mm})$ and PTV-C2 (CTV2 $+3 \mathrm{~mm})$.

\section{Radiation treatment planning}

All patients underwent IMRT for 6-MV photon external irradiation. The prescription doses and segmentation methods were as follows: PTV-G: dose was 66Gy in 30 fractions for stage T1 and T2; dose was 72-74 Gy in 32-33 fractions for stage $\mathrm{T} 3$ and T4; PTV-N: 66 Gy in 30 32 fractions; PTV-C1: 60 Gy in 30 32 fractions; PTV-C2: 54 Gy in 30 32 fractions.

\section{Chemotherapy}

The majority, i.e., 92\%, of patients underwent chemotherapy based on cisplatin, including induction, concurrent or adjuvant chemotherapy. The commonly used chemotherapy regimens include PF regimen (cisplatin $25 \mathrm{mg} / \mathrm{m}^{2} /$ day, for three days + fluorouracil $0.5 \mathrm{~g} / \mathrm{m}^{2} /$ day, for three days), TPF regimen (docetaxel $60 \mathrm{mg} / \mathrm{m}^{2} /$ day, for first day + cisplatin $25 \mathrm{mg} / \mathrm{m}^{2} /$ day, for three days + fluorouracil $0.5 \mathrm{~g} / \mathrm{m}^{2} /$ day, for three days), and GP regimen (gemcitabine $1 \mathrm{~g} / \mathrm{m}^{2} /$ day, for first and eighth day + cisplatin $25 \mathrm{mg} / \mathrm{m}^{2} /$ day, for three day). Concurrent chemotherapy was performed with cisplatin 80 $\mathrm{mg} / \mathrm{m}^{2} /$ day for three days, once every three weeks.

\section{Follow-up}

Follow-up time was defined as day one from treatment till death or the last followed up time. Patients were followed up once a week during radiotherapy to assess tumor regression and normal tissue adverse events. After the end of the treatment, the patients were followed up every 3 months for the first 2 years, and every 6 months from 3-5 years. After that, follow-up was performed once a year subsequently. Follow-up included physical examination, MRI or computer tomography (CT) of the nasopharynx, chest $\mathrm{CT}$, and abdominal ultrasound. Patients were subjected to cervical MRI or $\mathrm{CT}$, electronic nasopharyngoscopy and emission computed tomography (ECT) for bones if necessary. Imaging examinations were done 3 months after the end of radiotherapy and were performed every 6 months. After 5 years, the follow-up was carried out once every 1 year.

\section{Definition of failure mode}

The diagnostic criteria for recurrence included 
pathology, cytology, and imaging diagnosis. Patients with local or regional recurrence were screened, and MRI or CT was introduced for scanning the recurrence into the treatment planning system (TPS). The anatomical structure and the bony mark were used to fuse with pre-treatment CT, and the recurrence range $\left(\mathrm{V}_{\text {recur }}\right)$ was delineated. The treatment plan for the first-pass radiotherapy was replicated and calculated according to the original prescription dose. The recurrent lesions were evaluated by dosimetry. Evaluation indicators included $\mathrm{D}_{\max }, \mathrm{D}_{\min }, \mathrm{D}_{\text {mean }}$, and $\mathrm{V} 95 \%$ as received by $\mathrm{V}_{\text {recur. }}$ The failure modes were defined as follows: V95\% $\geq 95 \%$ is defined as recurrence in-field, $20 \% \leq$ V95\% $<95 \%$ for marginal failure, and V95\% < 20\% for out-field failure [18].

\section{Statistical analysis}

Kaplan-Meier analysis and log-rank tests using SPSS 23.0 (IBM Corporation, Armonk, NY) were used to present the survival data. Multivariate analysis was executed using the Cox risk regression model. $P$ values of less than 0.05 were considered to be statistically significant.

\section{Results}

\section{Patient characteristics and survival}

A total of 212 NPC patients were analyzed. The epidemiological properties of the patients, tumor staging and treatments were shown in Table 1 . There were 158 male patients and 54 female patients with a ratio of 2.9:1. The median age of the patients was 53.5 years (range 18-84 years). Histologically, most of the patients included had nonkeratinizing carcinoma, and only 4 patients had keratinizing squamous cell carcinoma. The overall staging of the patients was as follows: T1, 30 patients (14.1\%); T2, 68 patients (32.1\%) T3, 68 patients $(32.1 \%)$; and T4, 46 patients $(21.7 \%)$; N0, 5 patients $(2.4 \%)$; N1, 38 patients $(17.9 \%)$; N2, 130 patients $(61.3 \%)$ and N3, 39 patients $(18.4 \%)$; and stage I, 4 patients $(1.9 \%)$; stage II, 27 patients $(12.7 \%)$; stage III, 102 patients (48.1\%); stage IV, 79 patients (37.3\%). Follow-up time ranged from 4 months to 127 months, and the median follow-up of 34 months. The 5-year overall survival (OS) and local relapse-free survival (LRFS) were $77.8 \%$ and $85.6 \%$, respectively (Fig. 1A, 1B).

Age (Fig. 1C) and gender (Fig. 1D) showed no significant relationship with prognosis. The higher $\mathrm{T}$ stage (Fig. 1E), N stage (Fig. 1F), and AJCC stage (Fig. $1 G)$ showed negative correlation to OS by univariate analysis model. Further, multivariate Cox regression analysis of these factors was conducted and the results showed no significant correlation (Table 2).
Table 1. Patient characteristics

\begin{tabular}{|c|c|c|}
\hline Subject characteristics & Number of patients & $\%$ \\
\hline \multicolumn{3}{|l|}{ Age } \\
\hline$\leq 50$ & 94 & 44.3 \\
\hline$>50$ & 118 & 55.7 \\
\hline \multicolumn{3}{|l|}{ Gender } \\
\hline Female & 54 & 25.5 \\
\hline Male & 158 & 74.5 \\
\hline \multicolumn{3}{|l|}{ Histologic type } \\
\hline Keratinizing squamous cell carcinoma & 4 & 1.9 \\
\hline Nonkeratinizing carcinoma & 208 & 98.1 \\
\hline \multicolumn{3}{|l|}{ T stage } \\
\hline $\mathrm{T} 1$ & 30 & 14.1 \\
\hline $\mathrm{T} 2$ & 68 & 32.1 \\
\hline $\mathrm{T} 3$ & 68 & 32.1 \\
\hline $\mathrm{T} 4$ & 46 & 21.7 \\
\hline \multicolumn{3}{|l|}{ N stage } \\
\hline No & 5 & 2.4 \\
\hline N1 & 38 & 17.9 \\
\hline N2 & 130 & 61.3 \\
\hline N3 & 39 & 18.4 \\
\hline \multicolumn{3}{|l|}{ AJCC stage } \\
\hline I & 4 & 1.9 \\
\hline II & 27 & 12.7 \\
\hline III & 102 & 48.1 \\
\hline IV & 79 & 37.3 \\
\hline \multicolumn{3}{|l|}{ Radiotherapy } \\
\hline Median dose (Gy) & 70 & \\
\hline Range (Gy) & $66-74$ & \\
\hline \multicolumn{3}{|l|}{ Chemotherapy } \\
\hline No & 17 & 8.0 \\
\hline Induction & 12 & 5.7 \\
\hline Concurrent & 11 & 5.2 \\
\hline Induction+ Concurrent & 34 & 16.0 \\
\hline Concurrent+adjuvant & 51 & 24.1 \\
\hline Induction+ adjuvant & 24 & 11.3 \\
\hline Induction+ Concurrent+ adjuvant & 63 & 29.7 \\
\hline
\end{tabular}

Table 2. Multivariable Cox regression for analyzing the prognosis factors for NPC

\begin{tabular}{|c|c|c|c|c|}
\hline \multirow{2}{*}{$\begin{array}{l}\text { Subject } \\
\text { characteristics }\end{array}$} & \multicolumn{2}{|c|}{ No. of NPC patients } & \multirow[t]{2}{*}{$\mathrm{HR}(95 \% \mathrm{CI})$} & \multirow[t]{2}{*}{$P$} \\
\hline & overall & Dead $(\mathrm{N}, \%)$ & & \\
\hline \multicolumn{5}{|l|}{ Age } \\
\hline$\leq 50$ & 94 & $16(17.0)$ & 1 (Reference) & 1.0 \\
\hline \multirow[t]{2}{*}{$>50$} & 118 & $24(20.3)$ & $1.462(0.775-2.761)$ & \\
\hline & & & & 0.241 \\
\hline \multicolumn{5}{|l|}{ Gender } \\
\hline Female & 54 & $9(16.7)$ & 1 (Reference) & 1.0 \\
\hline \multirow[t]{2}{*}{ Male } & 158 & 31 (19.6) & $1.110(0.528-2.332)$ & \\
\hline & & & & 0.783 \\
\hline \multicolumn{5}{|l|}{ T stage } \\
\hline $\mathrm{T} 1$ & 30 & $1(3.3)$ & 1 (Reference) & 1.0 \\
\hline $\mathrm{T} 2$ & 68 & $10(14.7)$ & $3.783(0.484-29.563)$ & 0.205 \\
\hline $\mathrm{T} 3$ & 68 & $16(23.5)$ & $6.583(0.873-49.650)$ & 0.683 \\
\hline $\mathrm{T} 4$ & 46 & $13(28.3)$ & $1.270(0.194-74.403)$ & 0.028 \\
\hline \multicolumn{5}{|l|}{$\mathrm{N}$ stage } \\
\hline No & 5 & $0(0.0)$ & 1 (Reference) & 1.0 \\
\hline N1 & 38 & $1(2.6)$ & $1260.365(0.000-1.301 \mathrm{E} 83)$ & 0.939 \\
\hline N2 & 130 & $26(20.0)$ & $10518.969(0.000-1.075 \mathrm{E} 84)$ & 0.922 \\
\hline N3 & 39 & $13(33.3)$ & $18317.667(0.000-1.873 \mathrm{E} 84)$ & 0.917 \\
\hline \multicolumn{5}{|l|}{ AJCC stage } \\
\hline I & 4 & $0(0.0)$ & 1 (Reference) & 1.0 \\
\hline II & 27 & $0(0.0)$ & $1.001(0.000-1.021 \mathrm{E} 62)$ & 1.0 \\
\hline III & 102 & $17(16.7)$ & $2929.344(0.000-8442 \mathrm{E} 60)$ & 0.906 \\
\hline IV & 79 & $23(29.1)$ & $5885.555(0.000-1.696 \mathrm{E} 61)$ & 0.898 \\
\hline
\end{tabular}

Abbreviations: NPC, nasopharyngeal carcinoma; IQR, interquartile range. 


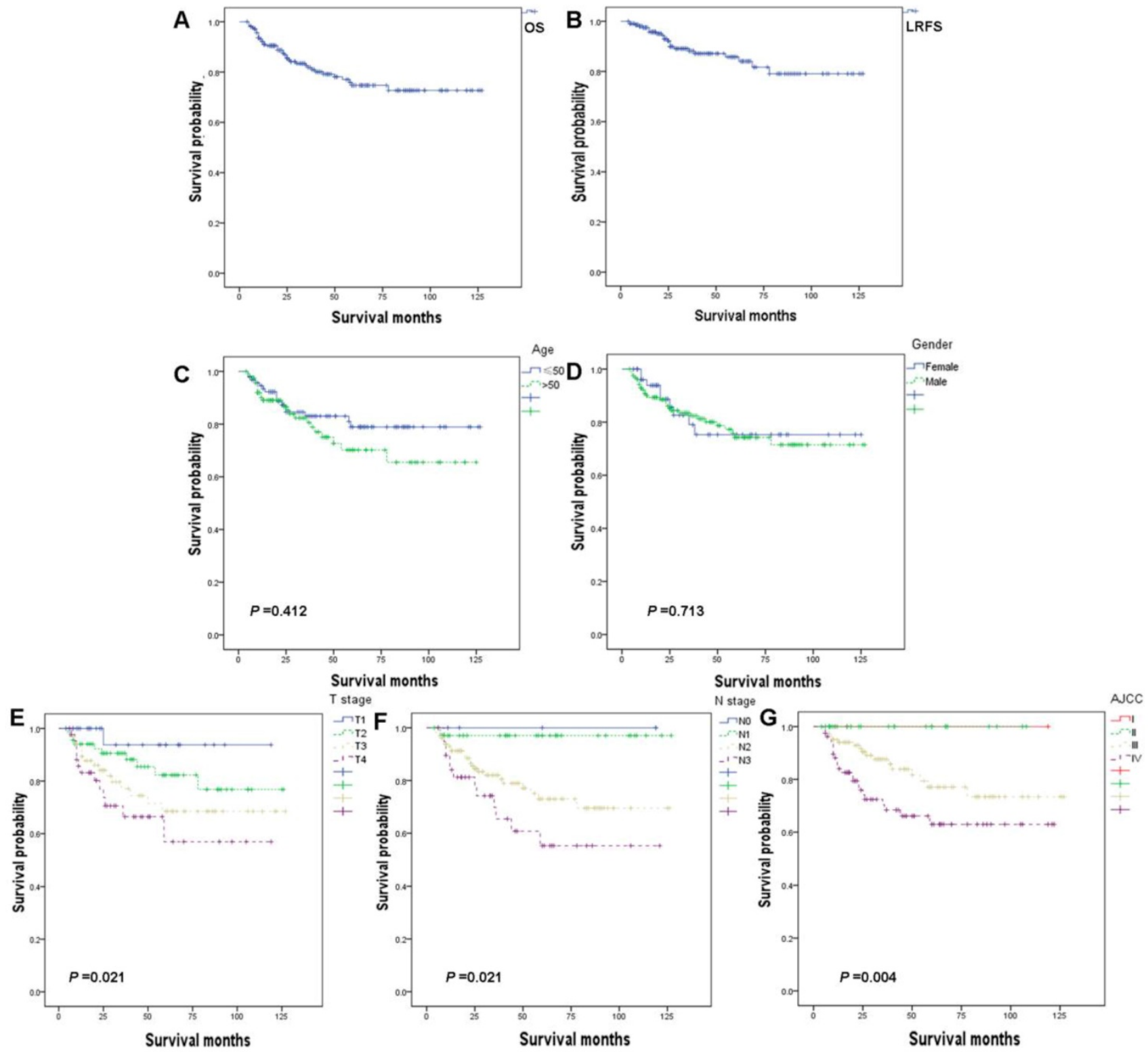

Figure 1. Kaplan-Meier analysis of overall survival (OS) (A) and local relapse-free survival (LRFS) (B) among patients, stratifed by age (C), gender (D), T stage (E), N stage $(\mathrm{F})$, and $\mathrm{AJCC}$ stage $(\mathrm{G})$.

\section{Rates of local-regional recurrence}

At the end of the follow-up period, localized recurrence occurred in 18 patients $(8.5 \%)$. Of these, $94.4 \%(17 / 18)$ patients were locally advanced patients, and most of them are in stages III and IV. The median time from the first treatment to recurrence was 24.5 months (range 7 to 73 months). Of these cases with recurrence, 11 of them were well rescued only through surgery (2), chemotherapy (3), RT (1), cervical lymph node dissection and RT (2), or RT combined with chemotherapy (3). Seven patients had unrecoverable local area recurrence, in which 1 patient had distant metastases before, during or after local recurrence, while 2 patients have refused any treatment.

\section{Dosimetric analysis of patients with recurrence}

The dose distribution of the target volumes in the first-pass radiotherapy of relapsed patients was shown in Table 3. Both GTV-P and GTV-N had higher dose coverage. Only $1.6 \%$ of the GTV-P and $0.5 \%$ of the GTV-N obtained $<95 \%$ of prescription dose. A greater part $(92.7 \%$ to $94.3 \%)$ of GTV-P and GTV-N obtained $\geq 100 \%$ of prescription dose. The doses of CTV1 and CTV2 used were similar. The mean doses of CTV1 and CTV2 were 64.3 and 55.7 Gy, respectively, and the $<95 \%$ prescription dose volumes were $0.9 \%$ and $0.6 \%$, respectively. 
Table 3. Dose-volume histograms (DVHs) statistics for patients of local-regional recurrence

\begin{tabular}{|c|c|c|c|c|}
\hline & GTV-P & GTV-N & CTV1 & CTV2 \\
\hline & Average (range) & Average (range) & Average (range) & Average (range) \\
\hline Dmax & 76.3 (72.1-78.4) & 73.7 (71.7-76.0) & 74.8 (69.9-78.2) & 62. (59.3-64.1) \\
\hline Dmean & $72.3(67.5-73.5)$ & $68.6(67.5-70.6)$ & $64.3(61.6-70.2)$ & 55.7 (53.2-58.1) \\
\hline Dmin & $57.3(50.8-64.4)$ & $57.1(50.1-65.3)$ & $42.9(22.6-58.5)$ & 40.7 (15.8-55.3) \\
\hline V95\% & $1.6(0-5.7)$ & $0.5(0-1.9)$ & $0.9(0-1.8)$ & $0.6(0-2.0)$ \\
\hline V100\% & 92.7 (79.3-98.5) & 94.3 (91.3-99.0) & 95.8(93.0-99.2) & 95.9 (92.4-98.9) \\
\hline V110\% & $0.2(0-0.9)$ & $0.6(0-2.6)$ & $0.9(0-3.7)$ & $2.2(0-5.6)$ \\
\hline
\end{tabular}

GTV-P gross tumor volume of primary tumor, GTV-N gross tumor volume of involved lymph nodes, CTV1 clinical tumor volume of the high-risk region, CTV2 clinical tumor volume of lymph nodal regions at low risk. Dmax = Maximum dose, Dmean $=$ Mean dose, Dmin $=$ Minimum dose. V95\% $=\%$ volume receiving $<95 \%$ of the prescribed dose, $\mathrm{V} 100 \%=\%$ volume receiving $>100 \%$ of the prescribed dose, $\mathrm{V} 110 \%=\%$ volume receiving $>110 \%$ of the prescribed dose.

Table 4. Details of local -regional recurrence patients

\begin{tabular}{|c|c|c|c|c|c|c|c|c|c|c|}
\hline \multirow{2}{*}{$\begin{array}{l}\text { No. of } \\
\text { Patient }\end{array}$} & \multirow{2}{*}{$\begin{array}{l}\text { Age } \\
\text { (years) }\end{array}$} & \multirow[t]{2}{*}{ Sex } & \multirow[t]{2}{*}{ Stage } & \multirow{2}{*}{$\begin{array}{l}\text { Site of } \\
\text { relapse }\end{array}$} & \multirow{2}{*}{$\begin{array}{l}\text { Location of the } \\
\text { recurrence volume }\end{array}$} & \multicolumn{4}{|c|}{ DVH statistics to recurrence volume } & \multirow{2}{*}{$\begin{array}{l}\text { Type of } \\
\text { relapse }\end{array}$} \\
\hline & & & & & & $\begin{array}{l}\text { Dmean } \\
(\mathrm{Gy})\end{array}$ & $\begin{array}{l}\text { Dmin } \\
(\mathrm{Gy})\end{array}$ & $\begin{array}{l}\text { Dmax } \\
\text { (Gy) }\end{array}$ & V95\% (\%) & \\
\hline 1 & 48 & Male & T2N2M0 III & Regional & CTV & 66.8 & 65.0 & 73.4 & 100 & In-field \\
\hline 2 & 54 & Male & T4N1M0 IV & Local & GTV & 65.6 & 62.1 & 76.9 & 100 & In-field \\
\hline 3 & 72 & Male & T1N2M0 III & Regional & CTV & 67.3 & 65.1 & 72.2 & 100 & In-field \\
\hline 4 & 79 & Male & T4N1M0 IV & Local & CTV & 64.5 & 60.6 & 71.3 & 100 & In-field \\
\hline 5 & 35 & Male & T4N0M0 IV & Local & Marginal to CTV & 62.5 & 55.1 & 70.3 & 90.2 & marginal \\
\hline 6 & 29 & Male & T2N2M0 III & Local & GTV & 70.2 & 65.7 & 77.5 & 100 & In-field \\
\hline 7 & 36 & Male & T1N2M0 III & Local & GTV & 67.3 & 61.4 & 75.8 & 98.4 & In-field \\
\hline 8 & 32 & Female & T2N2M0 III & Regional & CTV & 71.2 & 67.4 & 78.6 & 100 & In-field \\
\hline 9 & 35 & Male & T4N2M0 IV & Local & CTV & 65.5 & 62.0 & 74.2 & 100 & In-field \\
\hline 10 & 41 & Female & T1N3M0 IV & Local & GTV & 70.5 & 66.8 & 78.4 & 100 & In-field \\
\hline 11 & 52 & Male & T3N0M0 III & Local & CTV & 63.1 & 60.7 & 72.4 & 100 & In-field \\
\hline 12 & 84 & Female & T1N1M0 II & Local & CTV & 64.3 & 60.6 & 69.4 & 100 & In-field \\
\hline 13 & 49 & Female & T1N2M0 III & Local & CTV & 67.8 & 62.6 & 74.5 & 100 & In-field \\
\hline 14 & 70 & Female & T4N2M0 IV & Local & Marginal to CTV & 66.3 & 61.9 & 75.8 & 100 & marginal \\
\hline 15 & 52 & Male & T2N2M0 III & Local & Outside CTV & 64.7 & 59.3 & 72.0 & 99.6 & outside \\
\hline 16 & 70 & Male & T2N2M0 III & Local & CTV & 67.4 & 63.6 & 73.3 & 100 & In-field \\
\hline 17 & 81 & Female & T3N2M0 III & Local & CTV & 70.6 & 65.8 & 78.4 & 100 & In-field \\
\hline 18 & 55 & Male & T2N0M0 II & Local & GTV & 64.3 & 58.8 & 72.5 & 100 & In-field \\
\hline
\end{tabular}

\section{Local and regional failure mode}

Dosimetric analysis of recurrent lesions of these 18 patients showed that $83.3 \%$ of the recurrent lesions were in the $95 \%$ isodose curve and belonged to the in-field recurrence. Marginal failure and out-field failure accounted for $11.1 \%$ and $5.6 \%$, respectively (Table 4).

As shown in Table 4, the average minimum dose for the $\mathrm{V}_{\text {recur }}$ in the in-field recurrences was 63.2Gy (range 58.5Gy to 67.4Gy). The average mean dose was 67.1Gy (range 63.1Gy to 71.2Gy), and the average maximum dose was 74.6Gy (range 69.4Gy to 78.6Gy) for the $\mathrm{V}_{\text {recur }}$ in the in-field recurrences.

\section{Discussion}

IMRT has become the first choice for radiotherapy in patients with NPC. In recent years, various treatment centers have reported encouraging treatment results. Kwong et al. [19] have reported that 50 locally advanced patients had a local control rate of 95.7\% after 2 years of IMRT; Wang et al. [20] have revealed that 300 NPC cases who underwent IMRT had LRFS and OS of $94 \%$ and $86 \%$, respectively; and Lai et al. [21] have shown 5 years of LRFS and OS as $92.7 \%$ and $75.9 \%$, respectively. Kong et al. [7] have studied the survival of 370 NPC cases treated with IMRT, and the 2 -year OS was $94.1 \%$. In our study, the 5-year LRFS and OS were 85.6 and $77.8 \%$, respectively. Our results are similar to those reported in the above literatures, and long-term results need further observation.

Dosimetric analysis of recurrent lesions showed that the main recurrence pattern after IMRT treatment for patients with NPC was the in-field recurrence. With this, we hypothesized that most of the recurrences occur in high-dose areas, and so can the CTV range in some patients be reduced accordingly? This is because even in the large-scale and large doses of radiation, these recurrences cannot be avoided. Can we reduce the scope of CTV during the first radiotherapy to alleviate the side effects of radiotherapy in patients still remains a question. Salvage radiotherapy or surgery is performed again when relapsed in a small number of patients with recurrence. The radiotoxicity of NPC is very common 
and might be partly related to the non-standardized CTV. There are no predefined guidelines to direct the CTV and clinical outcomes. According to a study conducted by Sanford et al. [22], individualized CTV techniques were used to treat NPC patients and reduced the volume of CTV. Through long-term follow-up, high local control of the patients' lesions and low toxicity of normal organs were achieved. The treatment plan of this study included patients with nasopharyngeal lesions on only one side, and CTV only covers the ipsilateral parapharyngeal space to reduce the occurrence of closed jaw closure. CTV does not routinely include nasal, maxillary, sphenoid or ethmoid sinus. To reduce the dose to the mouth and soft palate, CTV does not cover the pharyngeal airway, reducing the incidence of mucositis or dry mouth. Combined with the data about the recurrence of our patients, individualized CTV was designed for patients who require radiotherapy in the future according to the actual situation of patients and CTV treatment range of Sanford et al., to minimize the side effects of radiotherapy.

Two patients in our study had a marginal recurrence. The first patient (Table 3, patient 5) had a relapse in the skull base and cavernous sinus, and had intracranial invasion before radiotherapy. The lowest dose of PTV-G was $57.4 \mathrm{~Gy}$, and $\mathrm{V}_{95 \%}$ was $9.2 \%$, suggesting that the patient had insufficient irradiation dose after first-time radiotherapy. In the second patient (patient 14 in Table 3), there was a recurrence of pterygopalatine fossa, and the patient had extensive skull base invasion before radiotherapy. The lowest dose of PTV-G was 65.8 Gy and $V_{95 \%}$ was $9.3 \%$ during the first-pass radiotherapy. We considered that the patient's recurrence was associated with the local low dose. The formation of local low-dose areas in the above two patients might be due to dose limitation of the optic nerve and optic chiasm. For patients with locally advanced, especially in stage T4, the dose of radiation therapy has become a problem in clinical treatment. The dose limitation has become an important factor for organ at risk (OAR), such as optic chiasm, optic nerve, brain stem, and so on, and so the local doses are difficult to reach the radical dose, even under the conditions of IMRT [23]. Some researchers have suggested that dose limitation of normal tissue (such as sacrificing the side of the optic nerve or temporal lobe) can be selectively reduced to achieve the goal by increasing the dose at the tumor target. However, whether the benefit of this method for patients with NPC is greater than the effect on quality of life still remains debatable.

The determination of the extent of CTV based on primary lesions plays a vital role in the control of local tumors as well as in the protection of normal tissues.
The current definition of CTV is mostly based on the experience with 3 dimensional or conventional radiation fields, which had a lack of individualization and different dose gradients. Even though the Radiation Therapy Oncology Group (RTOG) 0225 and 0615 [18] provided a reference for the CTV-1 range of NPC, the best definition of primary CTV has not yet been defined. In 2009, Liang et al. [24] have analyzed data from 943 NPC patients who underwent nasopharynx and neck MRI. After review by two radiologists, a low risk of bilateral tumors in the nasopharyngeal area $(10 \%)$ was detected. Local lesions gradually spread from the proximal part to the more distal part, while the local extension in a jumping pattern is uncommon. Thence, when the tumor erupts on the side of the nasopharynx, the high-risk bilateral anatomy should be covered by the CTV, while the moderate or low hazard and the contra lateral site ought to be ruled out from the CTV. Lin et al. [25] have analyzed data from 414 NPC patients who underwent IMRT treatment and defined CTV by GTV expansion of 5-10 mm margins in various paths, which included the whole nasopharyngeal mucosa and $5 \mathrm{~mm}$ submucosal volume. After follow-up for 5-years, the local recurrence showed no increase in the marginal correlation that is associated with the method used. For T4 disease, the flared edges of the six directions of the mass were notably smaller than those of the entire patient group. Hence, CTV-1, including GTV expansion of $5-10 \mathrm{~mm}$, the whole nasopharyngeal mucosa and a $5 \mathrm{~mm}$ sub mucosal volume was established. These results indicated that the target volume applied by the Fujian Medical University Cancer Hospital is sufficient. On the other hand, our organization used symmetrical coverage depicted by CTV and achieved good local control. By analyzing the location of the recurrence site, a guide to the CTV target volume was presented. According to our study, most of the anatomical sites are associated with low risk of bilateral tumors, and most of the local recurrence sites are located at the same site. We recommended that the contra lateral side of the tumor zone could be ruled out from the CTV.

CTV reduction can reduce the range of exposure to the contra lateral and mid-lower neck of the lesion, providing better protection to the OAR located in the area. Currently, even with IMRT, neck OAR might be subjected to varying degrees of damage. Acute and advanced dysphagia is a common complication after radiation therapy, with a total incidence of $18 \%-93.5 \%$ $[26,27]$. Symptoms of advanced dysphagia are associated with patients for many years [26], affecting the quality of life of the patients. The RTOG 0615 guidelines recommend an average dose of no more 
than 45 Gy for these OARs. Tiziana and his colleagues suggested that lessening the bulk of pharyngeal contractile muscles and larynx assists in receiving radiation doses of $\geq 60 \mathrm{~Gy}$. Further reduction of the volume of $\geq 50 \mathrm{~Gy}$ when possible is related to dysphagia and aspiration [28]. Similarly, radiation usually causes hypothyroidism (HT) and its incidence is $20.50 \%[29,30]$. If the thyroid gland receives radiation therapy dose of more than 45Gy, the occurrence of HT shows a significant increase [31]. Reducing CTV in the contra lateral neck region definitely reduces the dose of pharyngeal contractile muscles, esophagus, larynx, and carotid artery.

As displayed in Table 3, high local recurrences appeared in the high dose area. In our study, there was only one external field recurrence. The No.15 patient's stage was T2N2M0, and was accompanied by parapharyngeal space invasion. The first treatment included radiotherapy combined with concurrent chemotherapy, but the patient showed recurrence at 28 months after initial treatment. A retrospective study of pre-treatment MRI did not reveal any ethmoidal sinus disease. He survived till date (44 months) after receiving salvage chemotherapy and IMRT. Ng et al. [4] informed that one case had failure after one year of treatment, and the recurrence site was mainly located in the maxillary sinus and ethmoid sinus. The patient's stage was T3N2 without ethmoid sinus invasion before treatment. Is this likely to indicate another primary tumor? As the ethmoid sinus is a very rare site of NPC recurrence, it requires large number of samples and longer follow-up to determine if the posterior ethmoid ought to cover in high risk CTV.

However, our research has some limitations. Firstly, this study was a retrospective analysis with some bias. In the future, it is necessary to conduct randomized controlled clinical trials. Secondly, due to various factors such as time and location of the tumor, $100 \%$ accuracy of image fusion and dose analysis cannot be guaranteed. Thirdly, patients enrolled in this study underwent different chemotherapy regimens, showing varied impacts on the prognosis. Fourthly, this is a single-center data analysis, and multi-center clinical trials should be conducted in future to obtain more comprehensive data.

Our study analyzed local failure patterns after IMRT treatment in NPC patients. Recurrence in this field of research is the main mode of local recurrence, and a wide range of high doses of radiation cannot prevent local recurrence. Most of the local recurrence sites are located in the same site of the primary tumor, while bilateral tumors rarely occur. Therefore, we proposed that the range of the target volume of patients with NPC receiving IMRT treatment can be appropriately reduced.

\section{Acknowledgments}

Xiaojing Yang is the recipient of a grant from Shanghai Jiao Tong University Affiliated Sixth People's Hospital (contract grant number: ynlc201807), a grant from Shanghai Municipal Health Commission (20184Y0229) and a scientific research fund of Shanghai Sixth People's Hospital Medical Groups. Jie Fu is the recipient of a grant of Shanghai Shenkang Hospital Development Center Funds for Three Year Action Plan for Promoting Clinical Skills and Clinical Innovation Capacity in Municipal Hospitals (16CR3112B).

\section{Competing Interests}

The authors have declared that no competing interest exists.

\section{References}

1. Wang J, Shi M, Hsia Y, et al. Failure patterns and survival in patients with nasopharyngeal carcinoma treated with intensity modulated radiation in Northwest China: a pilot study. Radiat Oncol. 2012; 7: 2.

2. Ou X, Zhou X, Shi Q, et al. Treatment outcomes and late toxicities of 869 patients with nasopharyngeal carcinoma treated with definitive intensity modulated radiation therapy: new insight into the value of total dose of cisplatin and radiation boost. Oncotarget. 2015; 6: 38381-97.

3. Zhao $W$, Lei $H, Z$ hu $X$, et al. Investigation of long-term survival outcomes and failure patterns of patients with nasopharyngeal carcinoma receiving intensity-modulated radiotherapy: a retrospective analysis. Oncotarget. 2016; 7: 86914-25

4. Ng WT, Lee MC, Hung WM, et al. Clinical outcomes and patterns of failure after intensity-modulated radiotherapy for nasopharyngeal carcinoma. Int J Radiat Oncol Biol Phys. 2011; 79: 420-8.

5. Dawson LA, Anzai Y, Marsh L, et al. Patterns of local-regional recurrence following parotid-sparing conformal and segmental intensity-modulated radiotherapy for head and neck cancer. Int J Radiat Oncol Biol Phys. 2000; 46: 1117-26.

6. Li JX, Huang SM, Jiang $\mathrm{XH}$, et al. Local failure patterns for patients with nasopharyngeal carcinoma after intensity-modulated radiotherapy. Radiat Oncol. 2014; 9: 87.

7. Kong F, Ying H, Du C, Huang S, et al. Patterns of local-regional failure after primary intensity modulated radiotherapy for nasopharyngeal carcinoma. Radiat Oncol. 2014; 9: 60

8. Mao YP, Tang LL, Chen L, et al. Prognostic factors and failure patterns in non-metastatic nasopharyngeal carcinoma after intensity-modulated radiotherapy. Chin J Cancer. 2016; 35: 103

9. Lin S, Pan J, Han L, et al. Nasopharyngeal carcinoma treated with reduced-volume intensity-modulated radiation therapy: report on the 3-year outcome of a prospective series. Int J Radiat Oncol Biol Phys. 2009; 75: 1071-8.

10. Kiang A, Weinberg VK, Cheung $\mathrm{KH}$, et al. Long-term disease-specific and cognitive quality of life after intensity-modulated radiation therapy: a cross-sectional survey of nasopharyngeal carcinoma survivors. Radiat Oncol. 2016; 11: 127.

11. Zheng Y, Han F, Xiao W, et al. Analysis of late toxicity in nasopharyngeal carcinoma patients treated with intensity modulated radiation therapy. Radiat Oncol. 2015; 10: 17.

12. Lam TC, Wong FC, Leung TW, et al. Clinical outcomes of 174 nasopharyngeal carcinoma patients with radiation-induced temporal lobe necrosis. Int J Radiat Oncol Biol Phys. 2012; 82: e57-65.

13. Lin YS, Jen YM, and Lin JC. Radiation-related cranial nerve palsy in patients with nasopharyngeal carcinoma. Cancer. 2002; 95: 404-9.

14. Ratnasingam J, Karim N, Paramasivam SS, et al. Hypothalamic pituitary dysfunction amongst nasopharyngeal cancer survivors. Pituitary. 2015; 18: 448-55.

15. Low WK, Toh ST, Wee J, et al. Sensorineural hearing loss after radiotherapy and chemoradiotherapy: a single, blinded, randomized study. J Clin Oncol. 2006; 24: 1904-9.

16. Fang FM, Chiu HC, Kuo WR, et al. Health-related quality of life for nasopharyngeal carcinoma patients with cancer-free survival after treatment. Int J Radiat Oncol Biol Phys. 2002; 53: 959-68.

17. Ho S, Ip W, Chu S, et al. Treatment Regret in Long-term Survivors of Nasopharyngeal Carcinoma Treated with Radical Radiotherapy. Clin Oncol (R Coll Radiol). 2017; 29: e47. 
18. Li $\mathrm{Y}, \mathrm{Ou} \mathrm{X}$, Shen $\mathrm{C}$, et al. Patterns of local failures and suggestions for reduction of clinical target volume for nasopharyngeal carcinoma patients without cervical lymph node metastasis. Onco Targets Ther. 2018; 11: 2545-55.

19. Kwong DL, Pow EH, Sham JS, et al. Intensity-modulated radiotherapy for early-stage nasopharyngeal carcinoma: a prospective study on disease control and preservation of salivary function. Cancer. 2004; 101: 1584-93.

20. Wang $\mathrm{R}, \mathrm{Wu} \mathrm{F}$, $\mathrm{Lu} \mathrm{H}$, et al. Definitive intensity-modulated radiation therapy for nasopharyngeal carcinoma: long-term outcome of a multicenter prospective study. J Cancer Res Clin Oncol. 2013; 139: 139-45.

21. Lai SZ, Li WF, Chen L, et al. How does intensity-modulated radiotherapy versus conventional two-dimensional radiotherapy influence the treatment results in nasopharyngeal carcinoma patients? Int J Radiat Oncol Biol Phys. 2011; 80: 661-8.

22. Sanford NN, Lau J, Lam MB, et al. Individualization of Clinical Target Volume Delineation Based on Stepwise Spread of Nasopharyngeal Carcinoma: Outcome of More Than a Decade of Clinical Experience. Int J Radiat Oncol Biol Phys. 2019; 103: 654-68.

23. Orlandi E, Tomatis $\mathrm{S}$, Potepan $\mathrm{P}$, et al. Critical analysis of locoregional failures following intensity-modulated radiotherapy for nasopharyngeal carcinoma. Future Oncol. 2013; 9: 103-14

24. Liang SB, Sun Y, Liu LZ, et al. Extension of local disease in nasopharyngeal carcinoma detected by magnetic resonance imaging: improvement of clinical target volume delineation. Int J Radiat Oncol Biol Phys. 2009; 75: 742-50.

25. Lin S, Pan J, Han L, et al. Update report of nasopharyngeal carcinoma treated with reduced-volume intensity-modulated radiation therapy and hypothesis of the optimal margin. Radiother Oncol. 2014; 110: 385-9.

26. Balm AJ, Rasch CR, Schornagel JH, et al. High-dose superselective intra-arterial cisplatin and concomitant radiation (RADPLAT) for advanced head and neck cancer. Head Neck. 2004; 26: 485-93.

27. Levendag PC, Teguh DN, Voet P, et al. Dysphagia disorders in patients with cancer of the oropharynx are significantly affected by the radiation therapy dose to the superior and middle constrictor muscle: a dose-effect relationship. Radiother Oncol. 2007; 85: 64-73.

28. Rancati T, Schwarz M, Allen AM, et al. Radiation dose-volume effects in the larynx and pharynx. Int J Radiat Oncol Biol Phys. 2010; 76: S64-9.

29. Kumpulainen EJ, Hirvikoski PP, Virtaniemi JA, et al. Hypothyroidism after radiotherapy for laryngeal cancer. Radiother Oncol. 2000; 57: 97-101.

30. Diaz R, Jaboin JJ, Morales-Paliza M, et al. Hypothyroidism as a consequence of intensity-modulated radiotherapy with concurrent taxane-based chemotherapy for locally advanced head-and-neck cancer. Int J Radiat Oncol Biol Phys. 2010; 77: 468-76.

31. Bhandare N, Kennedy L, Malyapa RS, et al. Primary and central hypothyroidism after radiotherapy for head-and-neck tumors. Int J Radiat Oncol Biol Phys. 2007; 68: 1131-9. 